\title{
About the importance of being desulfated
}

\author{
Richa Khatri and Ernestina Schipani ${ }^{1}$ \\ Endocrine Unit, Massachusetts General Hospital-Harvard Medical School, Boston, Massachusetts 02114, USA
}

\begin{abstract}
Sulfated proteoglycans have important structural and signaling functions in the growth plate. In the October 1, 2008 , issue of Genes \& Development, Settembre and colleagues (pp. 2645-2650) report that lack of SUMF1, a crucial enzyme in the activation of sulfatases, causes a severe chondrodysplasia by augmenting fibroblast growth factor signaling and by hampering the autophagic process, which the investigators show is constitutively on in chondrocytes. The findings highlight the essential role of desulfation in cartilage biology and organogenesis.
\end{abstract}

Skeletal development depends on two mechanisms, intramembranous and endochondral (Karsenty and Wagner 2002; Karsenty 2003). The first, in which mesenchymal cells develop directly into osteoblasts, is involved in the formation of the flat bones of the skull. The second, accounting for the development of most other bones, involves a two-stage mechanism, whereby chondrocytes form a matrix template, the growth plate, which is then replaced by bone.

During endochondral bone development, growth plate chondrocytes undergo well-ordered and controlled phases of cell proliferation, maturation, and death. Proliferative chondrocytes synthesize collagen type II and form a columnar layer; they then stop proliferating and differentiate into post-mitotic hypertrophic cells. Hypertrophic chondrocytes express predominantly collagen type $\mathrm{X}$ and mineralize their surrounding matrix. This unique differentiation process is followed by death of hypertrophic chondrocytes, blood vessel invasion, and finally, replacement of the cartilaginous matrix with bone (Kronenberg 2003; Zelzer and Olsen 2003; Lefebvre and Smits 2005; Provot and Schipani 2005).

Chondrocytes are cells that produce and maintain a unique and abundant extracellular matrix. It is often stated that the size of an organ depends on two variables, cell number and cell size. This is only partially true for bone and cartilage, tissues in which matrix is as important as cells (at least from a quantitative point of view). The cartilaginous matrix is formed by two components, the proteoglycans and the collagens. Collagens provide structure and tensile strength to the matrix. There are three different types of collagens in the growth plate ma-

[Keywords: Proteoglycan; desulfation; FGF; chondrocytes]

${ }^{1}$ Corresponding author.

E-MAIL schipani@helix.mgh.harvard.edu; FAX (617) 724-7543.

Article is online at http://www.genesdev.org/cgi/doi/10.1101/gad.1735508. trix: fibrillar collagens, such as collagen type II and collagen type $\mathrm{XI}_{\text {; }}$ sheet-forming collagen, such as collagen type $\mathrm{X}_{\text {; }}$ and fibril-associated collagen, such as collagen type IX (Olsen 1996). Collagen type II is the most abundant collagen, and is produced by both proliferating and early hypertrophic chondrocytes. Collagen type $\mathrm{X}$ is exclusively expressed by hypertrophic chondrocytes. Collagen type IX binds glycosaminoglycans (GAGs). GAGs are long repeating polymers of specific disaccharides, which are often sulfated. Proteoglycans are macromolecules containing a core protein with multiple attached GAGs (Knudson and Knudson 2001). Because of their high content of GAGs, proteoglycans are highly hydrated. One of the most important extracellular proteoglycans is aggrecan, the predominant proteoglycan in cartilage. Aggrecan forms large aggregates, which give to cartilage its unique gel-like properties and its resistance to deformation. The central component of these aggregates is a long molecule of hyaluronan. Hyaluronan is the only extracellular oligosaccharide that is not covalently linked to a protein, as it is bound to aggrecan in a noncovalent fashion. This binding is facilitated by a link protein that binds to the aggrecan protein and to hyaluronan. The GAGs covalently attached to aggrecan are keratan sulfate and chondroitin sulfate. GAGs are not only structural components of the cartilaginous matrix, but they also play a critical role in signaling, and some of them are cell surface bound. In this regard, heparan sulfate proteoglycans (HSPGs) are particularly important (Farah-Carson et al. 2005). HSPGs are anionic molecules that consist of a protein core with covalently attached heparan sulfate (HS) chains of varying amounts and lengths. HS chains are long polymers of repeating $N$ acetylglucosamine and uronic acid disaccharides. Each single disaccharide can be further modified by sulfation by various sulfotransferases at the $N, 3-O$ or 6-O position of $\mathrm{N}$-acetylglucosamine, or the $2-\mathrm{O}$ position of uronic acid. These modifications enable HS chains to bind numerous proteins and act as coreceptors in growth factor signaling.

The fibroblast growth factor (FGF) family and their receptors (FGFRs) interact with cell surface HSPGs to coordinate cell-fate decisions. FGFs are a family of polypeptides that have critical roles in cell growth, differentiation, survival, and development (Ornitz and Marie 2002). They can activate one of four high-affinity FGFRs. The importance of FGF signaling in skeletal development was first revealed with the discovery that a point 
mutation in the transmembrane domain of FGFR3 leading to its constitutive activation is the etiology of Achondroplasia, one of the most common and severe forms of dwarfism (Vajo et al. 2000). Since this discovery, the etiology of many other human skeletal dysplasias has been attributed to specific mutations in the gene encoding FGFR3. Deletion of this receptor in mice causes increased chondrocyte proliferation and an expanded growth plate (Ornitz and Marie 2002). Conversely, increased activity of FGFR3 leads to reduced chondrocyte proliferation and dwarfism, as in humans (Ornitz and Marie 2002). FGF18, a ligand produced by the perichondrium that surrounds the growth plate, is involved in endochondral ossification by regulating growth plate chondrocyte proliferation, hypertrophy, and cartilage vascularization. It binds and activates FGFR3 with high affinity. This binding is facilitated by HSPGs. Upon binding, the receptor homodimerizes and initiates its autophosphorylation and stimulation of its tyrosine kinase activity. Mice that are null for FGF18 have a growth plate phenotype that closely resembles what is observed in the FGFR3-null mice; FGF18 is thus thought to be the critical ligand for FGFR3 in growth plate chondrocytes (Liu et al. 2002; Hinoi et al. 2006). There have been many proposed models to describe the interaction of HS with FGFs and their receptors, yet the precise molecular detail is still being determined. Nevertheless, FGF signaling requires HSPGs in order to form stable ligand/receptor complexes by likely protecting the FGF ligand from proteolytic degradation and by enhancing and stabilizing cell surface ligand/receptor interactions (Ornitz and Marie 2002; Jackson et al. 2006).

Sulfation is thus clearly an important modification that is necessary for matrix composition and signaling in the growth plate. However, it is also important for sulfated molecules to be degraded by sulfatases. In the October 1, 2008. issue of Genes \& Development, by using tools provided by mouse genetics, Settembre et al. (2008a) provide solid and strong evidence that SUMF1, a crucial enzyme in activation of sulfatase, has an essential role in cartilage development by regulating both autophagy and Fgf18- dependent modulation of chondrocyte proliferation. These novel findings are important and exciting for numerous reasons. The analysis of the mouse reported in this study provides, for the first time, a mechanistic understanding of the severe skeletal abnormalities observed in humans who carry loss-of-function mutations of SUMF1. Moreover, the study further proves that sulfation is a crucial event in modulating in vivo activity of the FGF family of proteins, in particular FGF18. Lastly, and more importantly, this study is the first in vivo demonstration that autophagy is a critical survival mechanism for growth plate chondrocytes, and it provides evidence that this survival mechanism is constitutively on in chondrocytes.

\section{Sulfatases, SUMF1, and FGFs}

Sulfatases are enzymes that hydrolyze sulfate esters from many substrates, including GAGs, sulfolipids, and steroid sulfates (Sardiello et al. 2005). In vertebrates, these hydrolases are associated with the turnover and degradation of molecules that are hydrolyzed in lysosomes in conjunction with acidic glycosidases. Sulfatases contain a highly conserved amino acid sequence, implying that they have evolved from a common ancestral gene. They share a common mechanism of sulfate ester hydrolysis in both prokaryotes and eukaryotes. In prokaryotes, sulfatases are sulfur scavengers, while in eukaryotes they play a key role in the degradation of molecules such as GAGs. Eukaryotic sulfatases have a common cysteine residue as part their active site. This residue is post-translationally converted to $\mathrm{C}_{\alpha}$-formylglycine (FGly) in the endoplasmic reticulum (ER), before the sorting of sulfatases to different cellular localizations. FGly is indispensable for the catalytic activity of sulfatases.

The role of sulfatases in human metabolism has been outlined by the study of inherited diseases due to sulfatase deficiencies, including the rare autosomal recessive disorder Multiple-Sulfatase Deficiency (MSD). In MSD, there is a severe reduction of all sulfatase activity due to mutations of the Sulfatase-Modifying Factor 1 gene (SUMF1) (Cosma et al. 2003; Dierks et al. 2003; Zito et al. 2007). This gene encodes for the FGly-generating enzyme (FGE) responsible for the post-translational conversion of cysteine to FGly. To this end, sulfatases appear to be the only target of SUMF1. A paralog of SUMF1, SUMF2, has been cloned recently. Both SUMF1 and SUMF2 are proteins localized in the ER and, interestingly, SUMF2 is an inhibitor of the enhancing effect of SUMF1 on the sulfatases (Zito et al. 2007). In MSD the activity of all sulfatases is severely reduced due to the inability to generate the FGly residues. Patients with MSD thus have a phenotype that encompasses the features of disorders caused by single-sulfatase deficiency, such as metachromatic leukodystrophy mucopolysaccharidoses, X-linked ichythyosis, and chondrodysplasia punctata. Mice lacking SUMF1 as a result of homologous recombination phenotypically reproduce numerous clinical features of patients with MSD (Settembre et al. 2007). They show early postnatal lethality, skeletal deformities, growth retardation, and severe neurological degeneration. Notably, sulfatase activity is completely absent in mice lacking SUMF1, which indicates that this protein is indeed the critical and nonredundant regulator of sulfatase activity in mammals. In the study published in the October 1, 2008, issue of Genes \& Development, Settembre et al. (2008a) now provide very elegant evidence that the skeletal abnormalities of SMUF1 $1^{-/-}$mice are in part caused by an exaggeration of FgG18 signaling. In particular, removal of one allele of Fgf18 was able to rescue both the reduced bone length, the impaired chondrocyte proliferation, and the abnormal collagen accumulation observed in SUMF1 ${ }^{-/-}$mice. Furthermore, the investigators provide strong in vitro evidence of increased FGF activity in chondrocytes lacking SUMF1. All in all, the study proves that sulfation is a crucial event in modulating the in vivo activity of the FGFs, and in particular of FGF18. 


\section{Sulfatases, SUMF1, and autophagy}

Interestingly, lack of one allele of FGF18 was not able to rescue the decreased cellularity of SUMF1 ${ }^{-/-}$growth plates. Settembre et al. (2008a) show that this hypocellularity is secondary to an impairment of autophagy, which is independent of FGF18 activity. In general, the degradation of intracellular proteins is performed by two mechanisms: the ubiquitin-proteosome system and autophagy. The process of autophagy is lysosomal dependent and activated in numerous stress conditions. Autophagy, from the Greek "phagy" (to eat) and "auto" (oneself), is a process by which cells digest their own cytoplasm and organelles in order to provide macromolecules for energy generation, and thus survive a variety of stresses (Levine and Klionsky 2004; Shintani and Klionsky 2004; Jin and White 2008). The word was first used by deDuve, who discovered the lysosomes, and also provided the first evidence that lysosomes are involved in autophagy (Klionsky 2008). Autophagy is linked to a variety of health and disease conditions, including development and differentiation, cancer, tissue degeneration, and infections. The identification of a specific and evolutionary conserved set of genes involved in autophagy has opened new doors to the understanding of autophagy in such a variety of biological processes. Autophagy can be differentiated in "microautophagy" and "macroautophagy" (Levine and Klionsky 2004). Microautophagy is the direct engulfment of cytoplasm by the lysosomes; macroautophagy refers to the formation of a doublemembrane vesicle containing portions of cytoplasm, which subsequently fuses to the lysosome. Autophagy is essential in mitochondria homeostasis; moreover, the autophagic process, which is also triggered by ER stress, is required to counterbalance the expansion of ER during the unfolded protein response (UPR) (Bernales et al. 2006; Yorimitsu et al. 2006; Ding et al. 2007; Yorimitsu and Klionsky 2007). Prolonged autophagy may lead to cell death. Autophagy is thus a double-edged sword, as it can promote either survival or death depending on the specific biological setting (Shintani and Klionsky 2004).

Lysosomal storage disorders (LSDs), which are caused by deficiencies of lysosomal hydrolases, can lead to abnormalities of the autophagic process. Settembre et al. (2008b) have reported previously that MSD, which, as aforementioned, is caused by the deficiency of SUMF1 gene, determines cellular dysfunction by blocking autophagic protein clearance. This is due to an impairment of autophagosome-lysosome fusion, resulting in an accumulation of autophagosomes. Consistent with these findings, lack of SUMF1 leads to massive vacuolization, intracellular accumulation of GAGs in numerous cell types, and block of autophagy (Settembre et al. 2008b).

In the October 1, 2008, issue of Genes\& Development, Settembre et al. (2008a) now provide, for the first time, in vivo evidence that the autophagic pathway is constitutively on in growth plate chondrocytes, and show that this pathway is severely hampered in chondrocytes lacking SUMF1. This likely leads to cell death, thus causing the severe hypocellularity that is observed in SUMF1 $1^{-/-}$ growth plates. Notably, no cytoplasm vacuolization could be detected in SUMF1 ${ }^{-/-}$osteoblasts, which indicates that desulfation is not a critical step in the biology of osteoblasts, different from what occurs in chondrocytes.

\section{Autophagy and chondrocytes}

In general, autophagy is particularly important under metabolic stress conditions. In particular, an increasing body of evidence has shown that hypoxia may trigger autophagy, with mechanisms that may or may not involve the transcription factor Hif- $1 \alpha$ and its target gene BNIP3 (Tracy et al. 2007; Azad et al. 2008; Papandreou et al. 2008; Zhang et al. 2008). The hypoxic stress has been also involved in the UPR (Feldman et al. 2005), which could be an additional mechanism adopted by hypoxia to promote autophagy, even if, to this end, no experimental link has been provided between UPR and autophagy in hypoxic conditions.

Notably, the fetal growth plate is an avascular environment with a typical out-in gradient of oxygenation and a hypoxic central region (Schipani et al. 2001). The transcription factor Hif- $1 \alpha$ is a critical and nonredundant survival factor for hypoxic chondrocytes, as lack of Hif-1 $\alpha$ in the fetal growth plate leads to massive central cell death (Schipani et al. 2001). Recently, in vitro evidence in support of a role for autophagy in chondrocyte survival has been provided (Srinivas and Shapiro 2006).

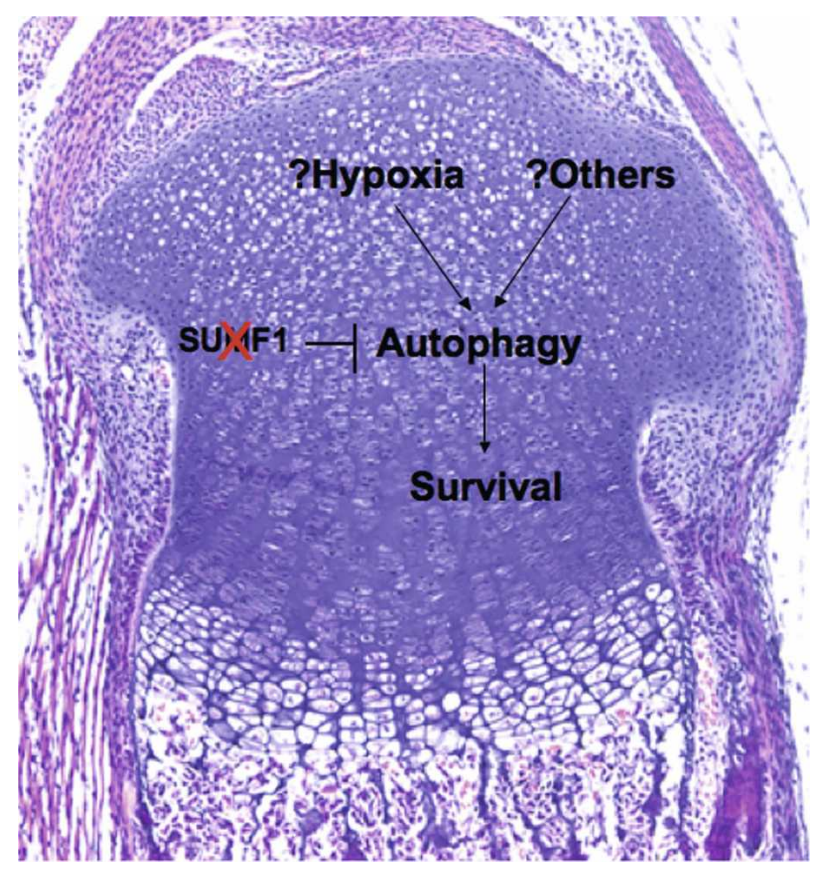

Figure 1. A hematoxylin and eosin $(\mathrm{H} \& \mathrm{E})$-stained histological section of a newborn proximal tibia growth plate is shown. Superimposed is a model of the role of autophagy in growth plate chondrocyte survival, particularly in the hypoxic region. According to this model, an impairment of the autophagic process, as occurs secondary to lack of SUMF1, would lead to cell death. 
Moreover, a relation between Hif- $1 \alpha$ and increased accumulation of autophagic proteins such as Beclin 1 has been documented, at least in vitro (Bohensky et al. 2007). Taken together, it is thus possible that one of the mechanisms adopted by Hif- $1 \alpha$ to allow survival of chondrocytes in a hypoxic environment could indeed involve positive modulation of the autophagic process. Consistent with this hypothesis, Settembre et al. (2008a) now provide elegant and important in vivo evidence that the autophagic process is constitutively on in growth plate chondrocytes.

Collectively, we can envision a model in which autophagy in growth plate chondrocytes would be required to support their survival, particularly in the hypoxic region, and thus an impairment of the process, as it occurs when proteoglycan desulfation is inhibited, would cause cell death (Fig. 1). Further experimental evidence in support of this model needs now to be provided. The study by Settembre et al. (2008a) is an important step forward in the understanding of both chondrocyte biology and the role of autophagy in survival and organogenesis.

\section{Acknowledgments}

This work was supported by NIH Grant RO1 AR048191-06 to E.S.

\section{References}

Azad, M., Chen, Y., Henson, E., Cizeau, J., McMillan-Ward, E., Israels, S., and Gibson, S. 2008. Hypoxia induces autophagic cell death in apoptosis-competent cells through a mechanism involving BNIP3. Autophagy 4: 195-204.

Bernales, S., McDonald, K., and Walter, P. 2006. Autophagy counterbalances endoplasmic reticulum expansion during the unfolded protein response. PLOS Biol. 4: e423. doi: 10.1371/journal.pbio.0040423.

Bohensky, J., Shapiro, I., Leshinsky, S., Terkhorn, S., Adams, C., and Srinvas, V. 2007. HIF-1 regulation of chondrocyte apoptosis: Induction of the autophagic pathway. Autophagy 3: 207-214.

Cosma, M., Pepe, S., Annunziata, I., Newbold, R., Grompe, M., Parenti, G., and Ballabio, A. 2003. The multiple sulfatase deficiency gene encodes an essential and limiting factor for the activity of sulfatases. Cell 113: 445-446.

Dierks, T., Schmidt, B., Borissenko, L., Peng, J., Preusser, A., Mariappan, M., and v Figura, K. 2003. Multiple sulfatase deficiency is caused by mutations in the gene encoding the human C- $\alpha$ formylglycine generating enzyme. Cell 113: 435-444.

Ding, W., Ni, H., Gao, W., Yoshimori, T., Stolz, D., Ron, D., and Yin, X. 2007. Linking the autophagy to biquitin-proteosome system is important for the regulation of endoplasmic reticulum stress and cell viability. Am. J. Pathol. 171: 513-524.

Farah-Carson, M., Hecht, J., and Carson, D. 2005. Heparan sulfate proteoglicans: Key players in cartilage biology. Crit. Rev. Eukaryot. Gene Expr. 15: 29-48.

Feldman, D., Chauhan, V., and Koong, A. 2005. The unfolded protein response: A novel component of the hypoxic stress response in tumors. Mol. Cancer Res. 3: 597-605.

Hinoi, E., Bialek, P., Chen, Y., Rached, M., Groner, Y., Behringer, R., Ornitz, D., and Karsenty, G. 2006. Runx2 inhibits chondrocyte proliferation and hypertrophy through its expression in the perichondrium. Genes \& Dev. 20: 29372942.

Jackson, R., Nurcombe, V., and Cool, S. 2006. Coordinated fibroblast growth factor and heparan sulfate regulation of osteogenesis. Gene 379: 79-91.

Jin, S. and White, E. 2008. Tumor suppression by autophagy through the management of metabolic stress. Autophagy 4: 563-566.

Karsenty, G. 2003. The complexities of skeletal biology. Nature 423: 316-318.

Karsenty, G. and Wagner, E. 2002. Reaching a genetic and molecular understanding of skeletal development. Dev. Cell 2: 389-406.

Klionsky, D. 2008. Autophagy revisited: A converation with Christian de Duve. Autophagy 4: 740-743.

Knudson, C. and Knudson, W. 2001. Cartilage proteoglycans. Semin. Cell Dev. Biol. 2001: 69-78.

Kronenberg, H. 2003. Developmental regulation of the growth plate. Nature 423: 332-336.

Lefebvre, V. and Smits, P. 2005. Transcriptional control of cell fate and differentiation. Birth Defects Res. C Embryo Today 75: 200-212.

Levine, B. and Klionsky, D. 2004. Development by self-digestion: Molecular mechanisms and biological functions of autophagy. Dev. Cell 6: 463-477.

Liu, Z., Xu, J., Colvin, J., and Ornitz, D. 2002. Coordination of chondrogenesis and osteogenesis by fibroblast growth factor 18. Genes \& Dev. 16: 859-869.

Olsen, B. 1996. Role of cartilage collagens in formation of the skeleton. Ann. N. Y. Acad. Sci. 8: 124-130.

Ornitz, D.M. and Marie, P.J. 2002. FGF signaling pathways in endochondral and intramembranous bone development and human genetic disease. Genes \& Dev. 16: 1446-1465.

Papandreou, I., Lim, A., Laderoute, K., and Denko, N. 2008. Hypoxia signals autophagy in tumor cells via AMPK activity, independent of Hif-1, BNIP3, and BNIP3L. Cell Death Differ. 15: 1572-1581.

Provot, S. and Schipani, E. 2005. Molecular mechanisms of endochondral bone development. Biochem. Biophys. Res. Commun. 328: 658-665.

Sardiello, M., Annunziata, I., Roma, G., and Ballabio, A. 2005. Sulfatases and sulfatases modifying factors: An exclusive and promiscuous relantionship. Hum. Mol. Genet. 14: 3203 3217.

Schipani, E., Ryan, H., Didrickson, S., Kobayashi, T., Knight, M., and Johnson, R. 2001. Hypoxia in cartilage: HIF-1 $\alpha$ is essential for chondrocyte growth arrest and survival. Genes \& Dev. 15: 2865-2876.

Settembre, C., Annunziata, I., Spampanato, C., Zarcone, D., Cobellis, G., Nusco, E., Zito, E., Tacchetti, C., Cosma, M., and Ballabio, A. 2007. Sytemic inflammation and neurodegeneration in a mouse model of multiple sulfatase deficiency. Proc. Natl. Acad. Sci. 104: 4506-4511.

Settembre, C., Arteaga-Solis, E., McKee, M.D., de Pablo, R., Al Awqati, Q., Ballabio, A., and Karsenty, G. 2008a. Proteoglycan desulfation determines the efficiency of chondrocyte autophagy and the extent of FGF signaling during endochondral ossification. Genes \& Dev. 22: 2645-2650.

Settembre, C., Fraldi, A., Jahreiss, L., Spampanato, C., Venturi, C., Medina, D., DePablo, R., Tacchetti, C., Rubinsztein, D., and Ballabio, A. 2008b. A block of autophagy in lyososomal storage disorders. Hum. Mol. Genet. 17: 119-129.

Shintani, T. and Klionsky, D. 2004. Autophagy in health and disease: A double-edged sword. Science 306: 990-995.

Srinivas, V. and Shapiro, I. 2006. Chondrocytes embedded in the 
epipheseal growth plates of long bones undergo autophagy prior to the induction of osteogenesis. Autophagy 3: 215216.

Tracy, K., Dibling, B., Spike, B., Knabb, J., Shumacker, P., and MacLeod, K. 2007. BNIP3 is an RB/E2F target gene required for hypoxia-induced autophagy. Mol. Cell. Biol. 27: 62296242.

Vajo, Z., Francomano, C., and Wilkin, D. 2000. The molecular and genetic basis of fibroblast growth factor receptor 3 disorders: The achondroplasia family of skeletal dysplasias, muenke craniosynostosis, and crouzon syndrome with acanthosis nigricans. Endocr. Rev. 21: 23-39.

Yorimitsu, T. and Klionsky, D. 2007. Eating the endoplasmic reticulum: Quality control by autophagy. Trends Cell Biol. 17: 279-285.

Yorimitsu, T., Nair, U., Yang, Z., and Klionsky, D. 2006. Endoplasmic reticulum stress triggers autophagy. J. Biol. Chem. 281: 30299-30304.

Zelzer, E. and Olsen, B. 2003. The genetic basis for skeletal diseases. Nature 423: 343-348.

Zhang, H., Bosch-Marce, M., Shimoda, L., Tan, Y., Baek, J., Wesley, J., Gonzalez, F., and Semenza, G. 2008. Mitochondrial autophagy is an Hif-1-dependent adaptive metabolic response to hypoxia. J. Biol. Chem. 283: 10892-10903.

Zito, E., Buono, M., Pepe, S., Settembre, C., Annunziata, I., Surace, E., Dierks, T., Monti, M., Cozzolino, M., Pucci, P., et al. 2007. Sulfatase modifying factor 1 trafficking through the cells: From endoplasmic reticulum to the endoplasmic reticulum. EMBO J. 26: 2443-2453. 


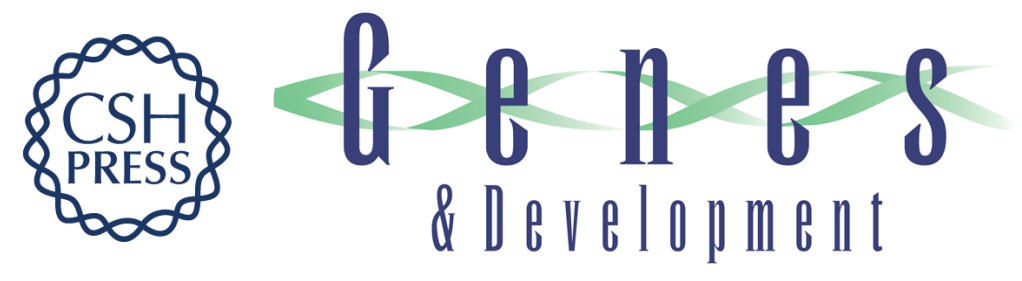

\section{About the importance of being desulfated}

Richa Khatri and Ernestina Schipani

Genes Dev. 2008, 22:

Access the most recent version at doi:10.1101/gad.1735508
Related Content Proteoglycan desulfation determines the efficiency of chondrocyte autophagy and the extent of FGF signaling during endochondral ossification Carmine Settembre, Emilio Arteaga-Solis, Marc D. McKee, et al. Genes Dev. October, 2008 22: 2645-2650

References This article cites 37 articles, 11 of which can be accessed free at: http://genesdev.cshlp.org/content/22/20/2750.full.html\#ref-list-1

Articles cited in:

http://genesdev.cshlp.org/content/22/20/2750.full.html\#related-urls

\section{License}

Email Alerting

Receive free email alerts when new articles cite this article - sign up in the box at the top

Service right corner of the article or click here.

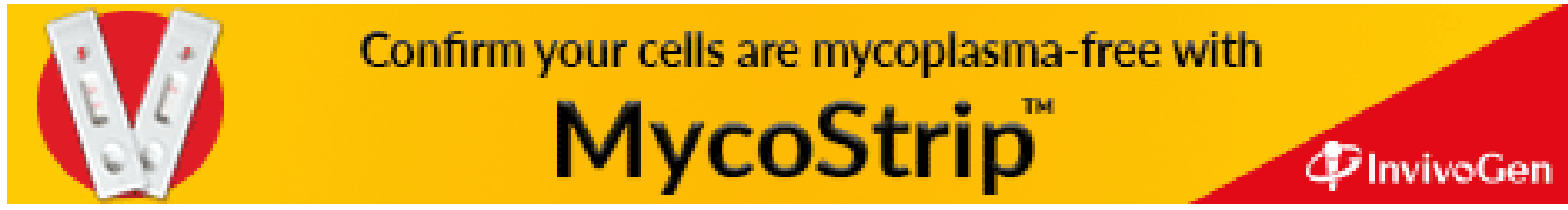

